\title{
Experimental Verification of the pumping of SIS mixer with an Distributed SIS Frequency Doubler
}

\author{
H. Rashid, V. Desmaris, A. Pavolotsky and V. Belitsky \\ Chalmers University of Technology, Gothenburg, Sweden
}

\begin{abstract}
In this paper, we present the performance of the first frequency multiplier using the non-linearity of distributed SIS junctions. The prototype doubler based on distributed SIS junction was for the first time able to pump an SIS mixer. The multiplication efficiency of the distributed SIS junction is $15-30 \%$ for a fractional bandwidth of $10 \%$ with excellent spectral line purity. The measured $-3 \mathrm{~dB}$ line width of the multiplied signal is better than $1 \mathrm{~Hz}$, which was limited by the resolution bandwidth of the spectrum analyzer. The results attained in this work show that the distributed SIS junction frequency multipliers have considerable future potential, and could possibly be used in LO source in single-end and multi-pixel SIS mixer receivers.
\end{abstract}

\section{INTRODUCTION}

$\mathrm{T}$ he extremely nonlinear behavior and quantum nature of operation of a Superconductor-Insulator-Superconductor (SIS) tunnel junction give reasons to believe that an SIS device designed for frequency multiplication can operate with very high conversion efficiencies, since the higher order harmonics of the quasiparticle tunnel current in an ideal SIS junction at zero physical temperature would be similar to the Fourier series of a Dirac delta function. However, until now, little work has been done on understanding, modeling and experimental verification of the harmonic generation (frequency multiplication) in SIS devices using quasiparticle tunneling effect [1],[2],[3]. Recently, we published an extensive paper treating the frequency multiplication possibilities and performance through theoretical modelling, which was experimentally verified [4],[5].

\section{LARGE Signal ModELling}

Accurate analysis of a circuit containing an SIS junction requires a self-consistent large-signal solution for the voltage across the nonlinear device. In the method used in [4], two realistic simplifications are made; $i$, The Josephson effect is not included as it is assumed to be suppressed with external magnetic field; ii. Self-oscillation and other nonharmonic frequency generation effects are ruled out.

The harmonic balance method employed follows the procedure described by Hicks et al. [6] for SIS mixers. The equivalent circuit diagram of the distributed SIS junction used in the analysis is shown in Fig. 1 [4]. The circuit diagram is bisected at the linear-nonlinear interface and each half is treated separately. The nonlinear portion of the circuit is modelled in the time domain whereas the embedding network, which includes the surface impedance and the geometrical capacitance of the distributed SIS junction, is treated in frequency domain.

Early results of the harmonic balance simulations show that the conversion efficiencies for the 2 nd harmonic at the pumping factor $\alpha=1.5$ and $\alpha=3$ attained with the large signal modeling are $90 \%$ and $50 \%$ respectively, details are found in [4]. The conversion efficiencies of the 3rd harmonic for the same pumping factors were approximately $30 \%$ and $15 \%$ respectively. Furthermore, according to the tunnel current spectrum attained by the large signal model and presented in Fig. 2, even higher harmonics such as the 4th or 5th may have sufficiently good multiplication efficiency, for the model based on the given above assumptions.

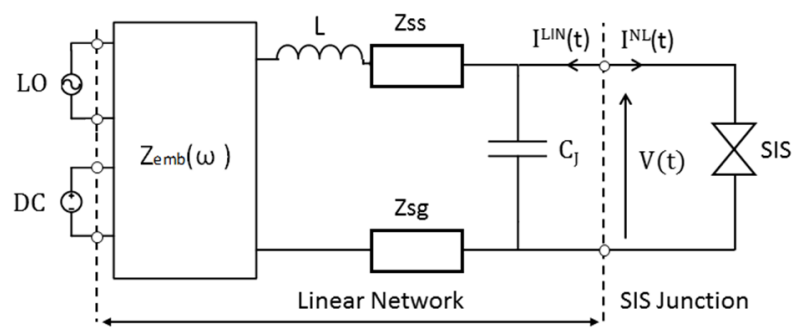

Fig. 1: Distributed SIS multiplier circuit. $\mathrm{L}$ and $\mathrm{C}_{\mathrm{J}}$ are geometrical inductance and capacitance, whereas Zss and Zsg are the surface resistance of the microstrip and ground electrode respectively.

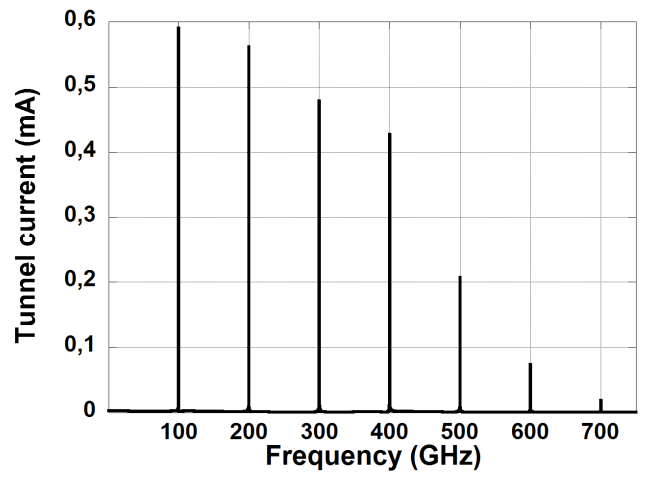

Fig.2: The large signal harmonic spectrum of the induced quasiparticle tunnel current. The pumping frequency signal at $100 \mathrm{GHz}$ is presented along with harmonic tones up to 7 th harmonic. It is clearly seen enhanced intensity of the harmonics $2,3,4$.

\section{MEASUREMENT RESUlts}

The modeling of the device shows that the distributed SIS junction can achieve high conversion efficiency, which was consistent with large signal harmonic balance simulations of the SIS junction multiplier [4].

The characterization of the device performance was carried out at $4 \mathrm{~K}$ in a cryostat with a closed-cycle refrigerator similar to [3]. Fig. 3 shows a block-diagram of the test setup used for the Distributed SIS frequency Multiplier (DSM) measurements. To study the response from DSM, an SIS mixer [7] that covers the RF frequencies from 163-211 GHz with 4$8 \mathrm{GHz}$ intermediate frequency (IF) bandwidth, was connected at the output of DSM . The DSM was pumped with a phase locked 83-105 GHz Gunn oscillator through a WR-10 waveguide, and the output of the DSM was coupled to the input 
of the mixer using a $3 \mathrm{~cm}$ long WR-5 waveguide. The SIS mixer was pumped using a 171-203 GHz LO consisting of an Agilent frequency synthesizer and followed by an amplifier / multiplier chain $(\times 6)[8]$. The IF output was analyzed with a spectrum analyzer.

In order to investigate the spectral response of the DSM, the LO frequency of the SIS mixer used for this purpose was set between 174-203 GHz, and the DSM was pumped with frequencies such that the expected DSM output power at the second harmonic would always fall inside the $4-8 \mathrm{GHz}$ band of the down converted mixer IF.

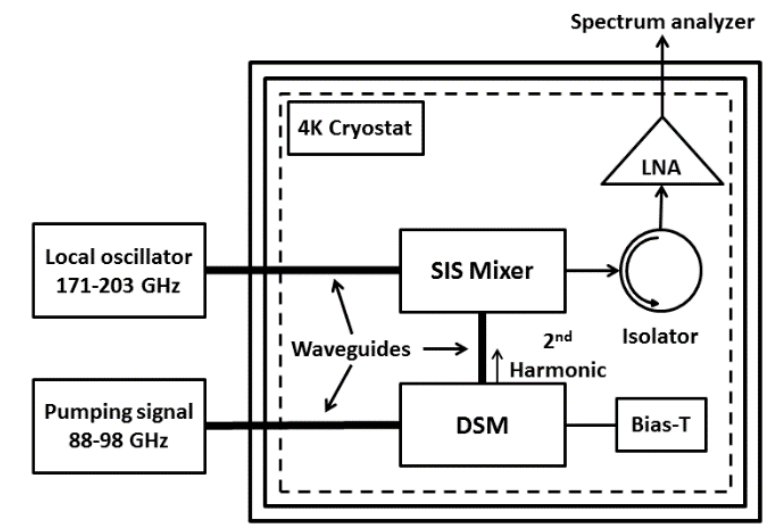

Fig. 3: Schematic of the experimental setup used to study the test device. The cryostat contains the DSM test chip connected through a WR-5 waveguide to a 163-211 GHz SIS mixer. Both the SIS mixer and the DSM test chip are pumped using two separate local oscillators

The measured output power of the distributed SIS junction in the regime of the generated second harmonic is in good agreement with the modeled output power. Furthermore, the distributed SIS junction as a frequency multiplier was for the first time able to pump an SIS mixer [4]. The efficiency of the distributed SIS junction is estimated to be $15-30 \%$ for a fractional bandwidth of $10 \%$ with excellent spectral line purity. The measured $-3 \mathrm{~dB}$ line width of the multiplied signal is $1 \mathrm{~Hz}$, which was limited by the lowest resolution bandwidth of the spectrum analyzer. The results presented in [4] show that the distributed SIS junction frequency multiplier has potential to become useful component in LO source of superconducting low-noise single-end receivers, SIS and HEB, and possibly be used in multi-pixel receivers.

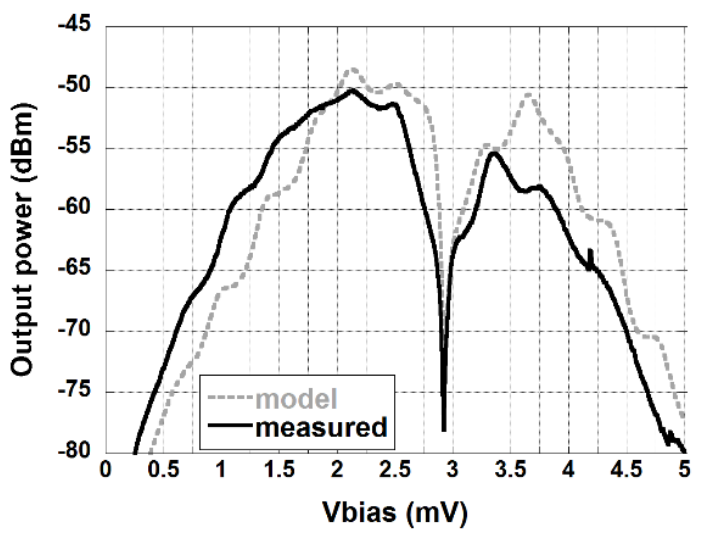

Fig.4: A comparison of the measured and predicted power at the 2nd harmonic i.e., $186 \mathrm{GHz}(93 \mathrm{GHz}$ input frequency) vs. bias voltage and pumping factor $\boldsymbol{\alpha} \approx 3$. The peak powers are due to the real part of current response function, i.e., IKK. The figure shows excellent correspondence between model and measurement. Furthermore, contribution of the IF amplification chain is removed here.

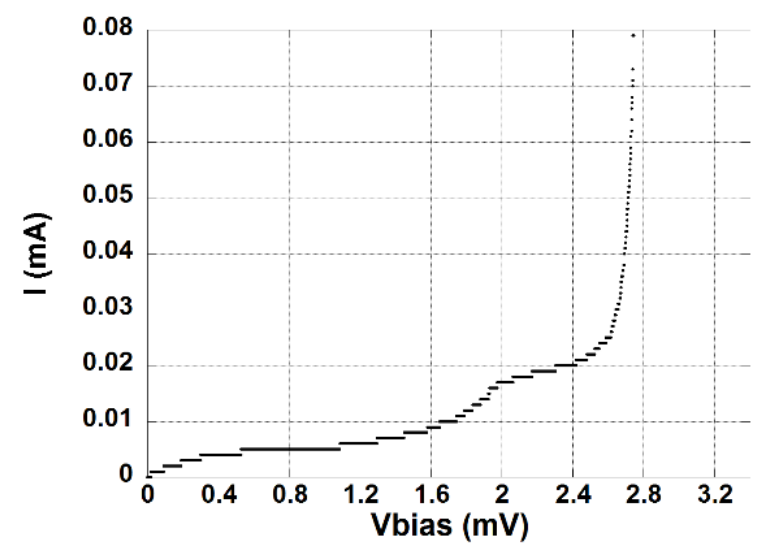

Fig.5: Pumped dc I-V characteristics of the SIS mixer. The zoomed plot clearly shows the quantum response of the SIS mixer for the output power of the DSM at twice the input frequency i.e., at $186 \mathrm{GHz}$. The step-structure of the zoomed IVC is due to limitation of data acquisition system (steps show the last digit of the current ADC).

\section{CONCLUSIONS}

In this paper, we presented the performance of the first frequency multiplier using the non-linearity of distributed SIS junctions. The prototype doubler based on distributed SIS junction was for the first time able to pump an SIS mixer. The multiplication efficiency of the distributed SIS junction is 15$30 \%$ for a fractional bandwidth of $10 \%$ with excellent spectral line purity. The measured $-3 \mathrm{~dB}$ line width of the multiplied signal is better than $1 \mathrm{~Hz}$, which was limited by the resolution bandwidth of the spectrum analyzer. The results attained in this work show that the distributed SIS junction frequency multipliers have considerable future potential, and could possibly be used in LO source in single-end and multi-pixel SIS mixer receivers.

\section{References}

H. D. Foltz and J. H. Davis, "Calculated performance of SIS junctions as frequency multipliers," in Microwave Symposium Digest, 1990., IEEE MTT-S International, 1990, pp. 307-310 vol.1. H. Rashid, V. Desmaris, A. Pavolotsky, and V. Belitsky, "Towards quantum limited frequency multiplication," in Infrared, Millimeter, and Terahertz waves (IRMMW-THz), 2014 39th International Conference on, 2014, pp. 1-2.

B. Billade, A. Pavolotsky, and V. Belitsky, "Experimental Study of Frequency Multiplication in a Distributed Array of SIS Junctions," Terahertz Science and Technology, IEEE Transactions on, vol. 4, pp. 254-259, 2014.

H. Rashid, S. Krause, D. Meledin, V. Desmaris, A. Pavolotsky, and V. Belitsky, "Frequency Multiplier Based on Distributed Superconducting Tunnel Junctions: Theory, Design and Characterization," IEEE Transactions on Terahertz Science and Technology, p. Accepted, 2016.

H. Rashid, V. Desmaris, A. Pavolotsky, and V.Belitsky, "Harmonic and Reactive Behavior of the Quasiparticle Tunnel Current in SIS Junctions," AIP Advances, 2016, Accepted.

R. G. Hicks, M. J. Feldman, and A. R. Kerr, "A general numerical analysis of the superconducting quasiparticle mixer," Magnetics, IEEE Transactions on, vol. 21, pp. 208-211, 1985.

B. Billade, A. Pavolotsky, and V. Belitsky, "An SIS Mixer With 2hf/k DSB Noise Temperature at 163 - $211 \mathrm{GHz}$ Band," Terahertz Science and Technology, IEEE Transactions on, vol. 3, pp. 416-421, 2013.

Virginia diodes, Inc. Available: http://vadiodes.com/ 